\title{
The probable role of insulin resistance and SIRT1 proteins in the Alzheimer's disease
}

\author{
Tural $\mathrm{R}^{1}$, Altan $\mathrm{N}^{1}$, Irkec $\mathrm{C}^{2}$, Sahin $\mathrm{D}^{1}$, Batur Caglayan $\mathrm{HZ}^{2}$ \\ Gazi University, Faculty of Medicine, Department of Medical Biochemistry, Ankara, Turkey. \\ rtural.2015@gmail.com
}

\begin{abstract}
OBJECTIVE: Recent evidence suggests that insulin resistance may play an important role in the pathogenesis of Alzheimer's disease (AD). In this study, the probable role of insulin resistance in the pathogenesis of AD was investigated in patients with Type 2 Diabetes Mellitus (T2DM).

METHODS: Serum amyloid beta $(A \beta)(1-42)$, insulin like growth factor-1 (IGF-1), sirtuin1 (SIRT1) and leptin protein levels were measured in serum samples of control $(n=26)$, probable $A D(n=26)$, and probable AD+T2DM patients $(n=12)$ using ELISA method. Mini mental state examination (MMSE) was performed to the patient and control groups.

RESULT: Serum IGF-1 significantly increased in the probable AD+T2DM group as compared to the control and probable $A D$ groups $(p<0.05)$. The levels of serum leptin significantly decreased in the probable $A D$ and $A D+T 2 D M$ groups as compared to the control $(p<0.05)$. There were no statistically significant differences in serum $A \beta(1-42)$ and SIRT1 levels among groups $(p>0.05)$.

CONCLUSION: The significant decrease in serum leptin levels in AD patients may indicate that it may be a therapeutic marker in AD. The level of serum A $\beta$ peptide and SIRT1 proteins can vary depending on the stage of the disease. Therefore, this study should be supported by more comprehensive studies in terms of the number of patients in advanced stage (Tab. 1, Fig. 4, Ref. 29). Text in PDF www.elis.sk KEY WORDS: Alzheimer disease, diabetes mellitus, insulin, IGF-1, amyloid beta (A $\beta$ ), sirtuin 1 (SIRT1), leptin.
\end{abstract}

\section{Introduction}

Alzheimer's disease (AD) is a neurodegenerative disease characterized by decreased cognitive function and memory. Amyloid plaques, histopathologic features of $\mathrm{AD}$, are composed of extracellular aggregates of amyloid beta $(A \beta)$ peptides (1). $A \beta(1-42)$ is the accumulating substance in the brain at early stages in all patients with Alzheimer's disease. Therefore, it is believed to be one of the key molecules in the pathology of Alzheimer's disease (2).

Diabetes mellitus (DM) is a systemic chronic metabolic disease associated with hyperglycemia, glycosuria, dyslipidemia, along with many other biochemical and clinical symptoms (3). Insulin resistance is known to play a crucial role in the pathogenesis of T2DM patients. T2DM and AD are becoming more common with age (4). Interestingly, recent evidence suggests that insulin resistance might also play an important role in the pathogenesis of $\mathrm{AD}$ (5). In T2DM induced animal models, insulin transport to the brain and neuronal insulin levels have decreased (4). These results

${ }^{1}$ Gazi University, Faculty of Medicine, Department of Medical Biochemistry, Ankara, Turkey, and ${ }^{2}$ Gazi University, Faculty of Medicine, Department of Neurology, Ankara, Turkey

Address for correspondence: R. Tural, Gazi University, Faculty of Medicine, Department of Medical Biochemistry, Ankara, Turkey

Ackonowledgement: This work was supported by Gazi University BAP \#01/2011-114. are consistent with reports of decreased insulin levels, decreased insulin receptor expression, and increased insulin resistance in the brains of $A D$ patients (4). In recent data which indicate that insulin resistance plays a role in the pathogenesis of patients with T2DM, it has been suggested that there is a relationship between T2DM and cognitive decline in terms of verbal memory (6). Long-term studies have shown that patients with T2DM have a 1.5-fold decrease in cognitive function as compared to those without diabetes (7).

IGF-1 regulates metabolic functions and growth in the brain, shows neurotrophic activity in the hippocampus in which learning and memory are involved and contributes to the survival of neurons. At the cellular level, impairment of IGF-1 uptake decreases neuronal resistance to $\mathrm{A} \beta$ peptide toxicity, increases cellular sensitivity to cell death signals, increases tau phosphorylation and ultimately leads to $A \beta$ accumulation in the brain (8). In addition to IGF-1, recent studies suggested that sirtuin proteins have diagnostic and therapeutic values for AD. SIRT1 is thought to have a neuroprotective effect against stress in cell cultures (9). Calorie restriction has been shown to mediate SIRT1 activity in protecting some animal models from neurodegenerative diseases, including AD $(10,11)$. Therapeutic potential of SIRT1 against AD has been shown in transgenic mice overexpressing SIRT1 (12). Although the role of SIRT1 in the prevention of AD is not precisely known, it has been proposed that the overexpression of ADAM10 gene encoding $\alpha$ secretase is associated with SIRT1 mediated protection against the accumulation of pathogenic $\mathrm{A} \beta$ peptide (9). It has 
been previously shown that autopsy samples of AD patients have a significant reduction in the concentration of parietal cortex SIRT1, and there is a strong correlation among tau accumulation, duration of symptoms and tissue SIRT1 concentration. However, their full relevance and role in the sequence leading to the development of $\mathrm{AD}$ are uncertain (13).

Recent studies have reported that leptin regulates $A \beta$ production and elimination in cell cultures and rodents (14). Improvement in cognitive performance was observed in mice treated with leptin (15). At the same time, it is also known that AD patients show weight loss and a decrease in circulating leptin levels (16). Greco et al (16), based on the known effects of insulin on tau phosphorylation and $\mathrm{A} \beta$ homeostasis, investigated whether leptin had similar activity. After leptin treatment, decreased phosphorylated tau levels were observed in vitro.

$\mathrm{AD}$ is the most common form of dementia and it is necessary to recognize the biochemical factors affecting the pathophysiology of this disease and identify new treatment strategies to reduce the incidence of the disease. In this study, it was aimed to investigate the probable role of insulin resistance in the pathogenesis of Alzheimer's disease by detecting A $\beta$ (1-42), IGF-1, SIRT1 and leptin levels in the serum samples of $\mathrm{AD}$ patients.

\section{Material and methods}

\section{Patients}

This study was approved by local ethics committee of our faculty of medicine. The study was carried out in patients who were followed up in the clinics of Gazi University Faculty of Medicine, Department of Neurology between 2011-2014. The study groups included 26 patients diagnosed with probable AD, 12 patients diagnosed with both probable AD and T2DM, 26 patients assigned as control without T2DM and probable AD. The mean duration of the disease was 4 years in patients with probable $\mathrm{AD}$ and with both probable AD and T2DM. Patients admitted to the neurology clinic due to forgetfulness and followed with the preliminary diagnosis of dementia (routine biochemistry, hemogram, radiographic examinations were performed) were included in the study. Patients who have previously been diagnosed with a cerebrovascular disease, Parkinson's disease, vascular dementia, vitamin B12 deficiency, and patients having dementia not associated with $\mathrm{AD}$ were excluded from the study.

\section{Probable Alzheimer's diagnosis}

During the evaluation for dementia associated with AD, NINCDS-ADRDA (National Institute of Neurological and Communicative Disorders, Stroke, Alzheimer's Disease, Related Diseases Association) (17) and DSM-IV (Diagnostic and Statistical Manual of Mental Disorders) (18) criteria were used in our study. Mini mental state examination (MMSE) was performed in patient and control groups. MMSE is a screening test consisting of 11 items grouped under five main categories, including orientation, registration memory, attention, mathematical operations, and remembrance and language (19). MMSE score between 24-30 points is considered normal, between 20-23 mild stage, between
10-19 middle stage, and a score between $0-9$ points is considered advanced stage dementia $(19,20)$. MMSE scores below 24 points were evaluated as cognitive impairment and have been further investigated.

\section{Biochemical analysis}

Routine tests were performed in central laboratory of Gazi University Hospital. In this study, $5 \mathrm{ml}$ of blood were taken into biochemistry tubes from all patient groups and serum samples were obtained and stored at $-80^{\circ} \mathrm{C}$ until analyses. TSH (uIU/ml), T3 (pg/ml), T4 (ng/dl), total cholesterol (mg/dl), HDL (mg/d), LDL (mg/dl), VLDL (mg/dl), vitamin B12 (pg/ml), fasting blood glucose (mg/dl), insulin (mU/L), Hbalc (\%) levels were measured using equipment in Central Laboratory of Gazi University Hospital (Roche HITACHI, COBAS e 601 (for TSH, T3, T4, vitamin B12 and insulin measurements); BECKMAN AU 2700 Plus (for total cholesterol, HDL, LDL, VLDL, fasting blood glucose measurements); Arkray ADAMS HA-8180V A1C Autoanalyser (for HbA1c measurement)).

Levels of Serum A $\beta$ (1-42) (Human amyloid beta peptide 1-42 (A $\beta$ 1-42) (EASTBIOPHARM, China)), IGF-1 (Human IGF-1 (AssayPro, St. Charles, USA)), SIRT1 (Human Sirtuin1(SIRT1) (EASTBIOPHARM)), and leptin (AssayPro AssayMax Human Leptin (AssayPro)) were determined by ELISA (Enzyme-Linked Immuno Sorbent Assay) method according to the instructions for use.

BioTek ELISA washer were used for the analysis of $A \beta$ (142) (ng/L), IGF-1 (ng/ml), SIRT1 (ng/ml) and Leptin (ng/ml) (VT, U.S.A.). For spectroscopic measurements, BioTek ELISA reader was utilized.

\section{Statistical analyses}

For the analysis of the obtained data, SPSS (Statistical Package for Social Sciences) version 15 was used. ANOVA (Tukey) test was performed for leptin, T3, total cholesterol, LDL, VLDL, vitamin B12, fasting blood glucose, insulin, HbA1c parameters; Kruskal-Wallis and then Mann-Whitney U tests were used for SIRT1, IGF-1, A $\beta$ (1-42), TSH, T4, HDL-cholesterol, MMSE nonparametric parameters. Values were presented as 'mean \pm standard deviation'. $\mathrm{p}<0.05$ was considered statistically significant.

\section{Results}

The average age and gender distribution of the patients in our study was found similar among study groups. The demographic values and blood biochemical values and statistical analyses are presented in Table 1 . Total cholesterol levels were significantly decreased in the probable $\mathrm{AD}+\mathrm{T} 2 \mathrm{DM}$ group compared to the control and probable $\mathrm{AD}$ groups $(\mathrm{p}<0.05)$; LDL levels were significantly reduced in the probable $\mathrm{AD}$ and $\mathrm{AD}+\mathrm{T} 2 \mathrm{DM}$ groups compared to the control group ; vitamin B12 levels were significantly increased in the probable $\mathrm{AD}$ group compared to the control group; fasting blood glucose, insulin, $\mathrm{HbA} 1 \mathrm{c}$ levels were increased significantly in the probable $\mathrm{AD}+\mathrm{T} 2 \mathrm{DM}$ group compared to the control and probable AD groups; MMSE levels were significantly decreased 
Tab. 1. The levels of thyroid hormones, serum lipids, vitamin B12, and fasting blood glucose, insulin, HbA1c, MMSE, age and sex among the groups.

\begin{tabular}{lccc}
\hline & Control $(\mathrm{n}=26)$ & AD $(\mathrm{n}=26)$ & AD+T2DM $(\mathrm{n}=12)$ \\
\hline TSH (uIU/ml) & $2.01 \pm 1.13$ & $1.72 \pm 0.72$ & $1.93 \pm 0.91$ \\
T3 (pg/ml) & $2.96 \pm 0.35$ & $2.88 \pm 0.37$ & $3.00 \pm 0.24$ \\
T4 (ng/dl) & $1.16 \pm 0.17$ & $1.22 \pm 0.19$ & $1.19 \pm 0.14$ \\
Total Cholesterol (mg/dl) & $218.15 \pm 28.88$ & $201.84 \pm 36.32$ & $162.58 \pm 30.47^{\mathrm{a}, \mathrm{b}}$ \\
HDL (mg/dl) & $46.92 \pm 6.43$ & $45.73 \pm 8.68$ & $41.25 \pm 7.91$ \\
LDL (mg/dl) & $145.34 \pm 20.07$ & $118.99 \pm 37.03^{\mathrm{a}}$ & $96.30 \pm 24.10^{\mathrm{a}}$ \\
VLDL (mg/dl) & $31.00 \pm 12.66$ & $25.21 \pm 10.70$ & $26.86 \pm 8.50$ \\
Vitamin B12 (pg/ml) & $329.38 \pm 112.20$ & $426.15 \pm 165.85^{\mathrm{a}}$ & $329.51 \pm 109.58$ \\
Fasting blood glucose(mg/dl) & $93.73 \pm 9.60$ & $99.88 \pm 11.07$ & $137.58 \pm 33.21^{\mathrm{a}, \mathrm{b}}$ \\
Insulin (mU/L) & $7.80 \pm 2.99$ & $8.01 \pm 3.47$ & $29.43 \pm 17.32^{\mathrm{a}, \mathrm{b}}$ \\
Hba1c (\%) & $5.69 \pm 0.23$ & $5.62 \pm 0.26$ & $7.29 \pm 1.57^{\mathrm{a}, \mathrm{b}}$ \\
MMSE (<24) & $25.84 \pm 3.30$ & $19.30 \pm 5.54 \mathrm{a}$ & $16.91 \pm 3.84^{\mathrm{a}}$ \\
Age & $70.92 \pm 7.05$ & $78.26 \pm 7.44$ & $72.83 \pm 10$ \\
Sex (Female/Male) & $18 / 8$ & $18 / 8$ & $5 / 7$ \\
\hline
\end{tabular}

AD: Alzheimer's disease, T2DM: Type 2 Diabetes Mellitus, MMSE: Mini mental state examination. ${ }^{\text {ap: }}$ Compared to the control group $\mathrm{p}<0.05$, ${ }^{\mathrm{b}} \mathrm{p}$ : Compared to the $\mathrm{AD}$ group $\mathrm{p}<0.05$

in the probable $\mathrm{AD}$ and $\mathrm{AD}+\mathrm{T} 2 \mathrm{DM}$ groups compared to the control group $(\mathrm{p}<0.05)$ (Tab. 1).

Serum IGF-1 levels were significantly increased in probable $\mathrm{AD}+\mathrm{T} 2 \mathrm{DM}$ group compared to the control and probable AD groups. Serum leptin levels were significantly reduced in

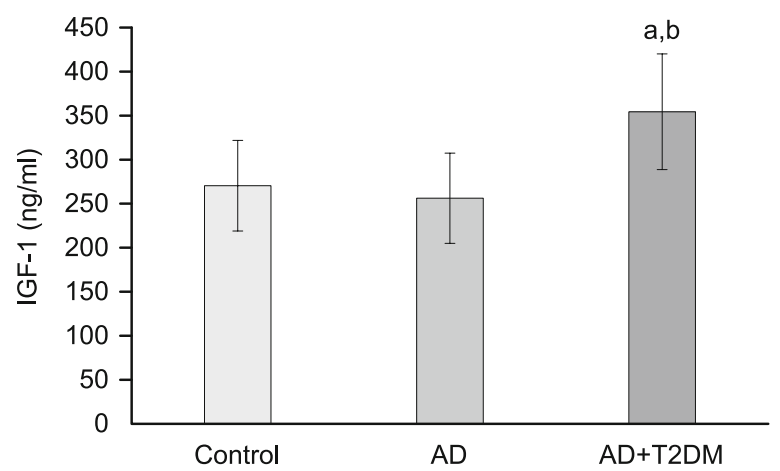

Fig. 1. IGF-1(Insulin like growth factor-1) concentrations of groups. AD: Alzheimer's disease, T2DM: Type 2 Diabetes Mellitus. ${ }^{\text {ap: }}$ Compared to the control group $\mathrm{p}<0.05$, ${ }^{\mathrm{b}} \mathrm{p}$ : Compared to the $\mathrm{AD}(\mathrm{Alz}-$ heimer's disease) group $\mathrm{p}<0.05$.

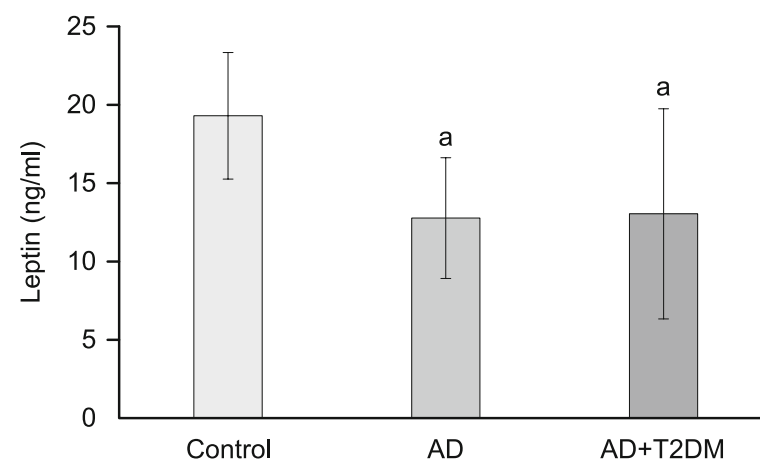

Fig. 2. Serum leptin concentrations among groups. AD: Alzheimer's disease, T2DM: Type 2 Diabetes Mellitus. a ${ }^{2}$ : Compared to the control group $\mathbf{p}<\mathbf{0 . 0 5}$. the probable $\mathrm{AD}$ and $\mathrm{AD}+\mathrm{T} 2 \mathrm{DM}$ groups compared to the control group $(\mathrm{p}<0.05)$ (Figs 1 and 2).

From the perspective of serum $A \beta$ (142) and SIRT1 levels, there was no statistically significant difference between groups ( $>0.05)$. (Figs 3 and 4).

\section{Discussion}

Epidemiological and clinical studies have indicated that there is an increased risk for $\mathrm{AD}$ in patients with insulin resistance. In these patients, abnormal insulin signalling and abnormal glucose use in the central nervous system (CNS) may trigger several metabolic disorders, contributing to the formation of neurofibrillary tangles and extracellular accumulation of $A \beta(21)$. It is reported that insulin inhibits tau phosphorylation and $A \beta$ accumulation in neurons by inhibiting GSK-3 $\beta$ (22). It is also known that insulin affects many brain functions, including cognition and memory (23). It is believed that in all patients with $\mathrm{AD}, \mathrm{A} \beta(1-42)$ is the key mole-

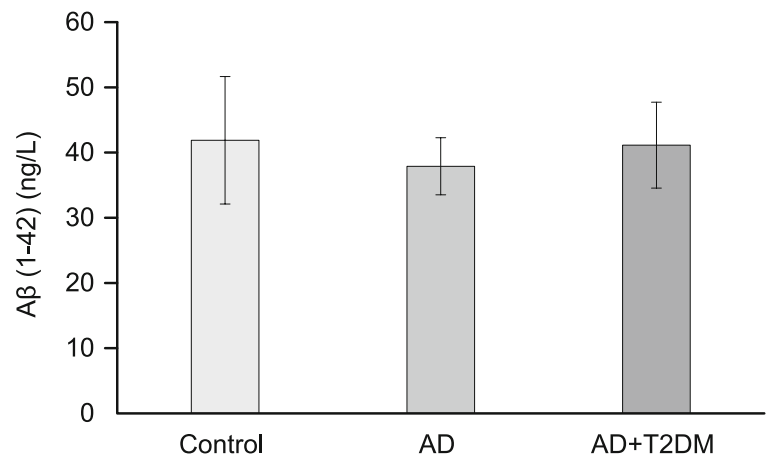

Fig. 3. Serum A $\beta$ (Amyloid beta peptides) (1-42) concentrations among groups. AD: Alzheimer's disease, T2DM: Type 2 Diabetes Mellitus. There was no statistically significant difference between groups $(p>0.05)$.

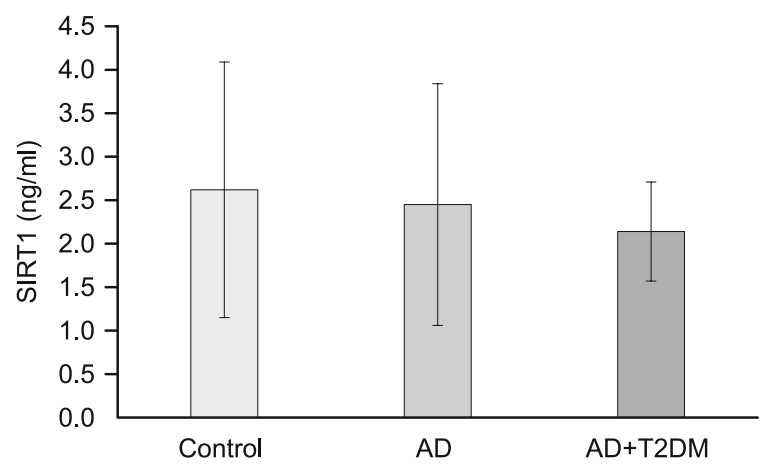

Fig. 4. SIRT1 (Sirtuin1) serum concentrations among groups. AD: Alzheimer's disease, T2DM: Type 2 Diabetes Mellitus. There was no statistically significant difference between groups $(p>0.05)$. 
cule in the pathology of AD because it is the first substance to accumulate in the brain (2).

IGF-I is a known angiogenic promoter (24). IGF-1 increases neuroprotective effects by increasing neuropil, increasing cognitive performance and decreasing IGF-1 level and together with aging leads to a decrease in brain size (24). It has been suggested that serum IGF-1 plays a role in the elimination of $A \beta$ proteins from the brain by increasing the entrance of $\beta$ A carrier proteins such as albumin, transthyretin and apolipoprotein $\mathrm{J}$ into the cerebrospinal fluid (CSF) (24). A $\beta$ proteins are then cleared from the blood with anti-A $\beta$ antibodies according to Jianping et al (25). In a study conducted by Yamamoto et al., low albumin-A $\beta$ complex has been associated with an increase in the prevalence of AD. It has been suggested that a decrease in albumin-dependent $A \beta$ in blood could be associated with a decrease in $A \beta$ excretion into the blood from the brain, and this situation may lead to the accumulation of $A \beta$ (1-42) in the brain (26). In our study, because of increased insulin resistance in the probable $\mathrm{AD}+\mathrm{T} 2 \mathrm{DM}$ group, the accumulation of $\mathrm{A} \beta$ may tend to increase slightly in this group compared to the $\mathrm{AD}$ group. According to our results, the accumulation of $A \beta$ (1-42) protein levels, which may occur in diabetic patients due to insulin resistance, may be improved due to increased IGF1 level in these patients. However, our results may also indicate that $A \beta$ proteins may have been cleared from blood by $\mathrm{A} \beta$ antibodies, consistent with the results of a study by Jianping et al (25).

It has been shown that leptin increases apoE-mediated $\mathrm{A} \beta$ uptake into the cell and decreases the extracellular concentrations of $A \beta$ in the brain. Leptin and insulin act dose-dependent and synergistically to reduce hyperphosphorylation of Tau, a key component of NFYs, which are major histopathologic features of $\mathrm{AD}$ (27). Chronic leptin therapy has been shown to improve memory performance in transgenic animal models with AD (28). In the study of Bigalke et al (29), plasma leptin levels were found significantly lower in the patients with early $\mathrm{AD}$ compared to the control group. A decrease in the circulating leptin in these individuals was associated with cognitive dysfunction. Consistent with this, in our study, both the probable $\mathrm{AD}$ and $\mathrm{AD}+\mathrm{T} 2 \mathrm{DM}$ groups were showed to have significantly decreased serum leptin levels and the MMSE score compared to the control group.

SIRT1 proteins have been reported to play a role in the prevention of brain degeneration in $\mathrm{AD}$. $\alpha$-secretase is responsible for the non-amyloidogenic cleavage of the amyloid precursor protein. SIRT1 increases the expression of the ADAM10 gene which encodes $\alpha$-secretase. It is thus effective in protecting against pathogenic $\mathrm{A} \beta$ peptide accumulation (9). In a study performed by $\mathrm{Ku}-$ mar et al (9), a significant decrease in serum SIRT1 concentration was observed in individuals with AD compared to healthy elderly individuals. In addition, serum SIRT1 concentration was found significantly lower in healthy elderly compared to young healthy controls. In the study of Julien et al (13), a significant decrease in SIRT1 was reported in postmortem examination of parietal cortex of patients with $\mathrm{AD}$. However, when a second cohort analysis was performed according to the antemortem clinical diagnosis, it was found that there was a decrease in SIRT1 in the cortex of individuals with $\mathrm{AD}$, but this decrease was not statistically significant and the individuals with mild cognitive impairment and no cognitive impairment were very similar to the SIRT1 concentrations. These results suggest that clinical diagnosis and postmortem evaluations may differ in patients with AD and SIRT1 measurements are unlikely to be a marker of value for early stages of AD or clinically diagnosed AD patients. Consistent with this study, in our study, although there was a decrease in serum SIRT1 levels in patients with $\mathrm{AD}$, no significant difference was found between the groups.

\section{Conclusion}

In our study, serum leptin level was decreased in patients with probable $\mathrm{AD}$ and probable $\mathrm{AD}+\mathrm{T} 2 \mathrm{DM}$. It can be considered as therapeutic marker in patients with $\mathrm{AD}$, but did not make the difference to be a therapeutic marker in diabetic patients with AD. Serum $A \beta$ peptide and SIRT1 levels were not helpful in diagnosing the early stage of probable AD + T2DM. The cause of this condition may be related to the stage of the disease. Therefore, the effects of serum A $\beta$ peptide and SIRT 1 proteins levels in Alzheimer's disease should be supported by more comprehensive studies in terms of the number of patients and with long-term follow-up.

\section{References}

1. Pohanka M. Oxidative Stress in Alzheimer Disease as a Target for Therapy. Bratisl Med J 2018; 119 (9): 535-543.

2. Kawarabayashi T, Shoji M. Plasma Biomarkers of Alzheimer's Disease. Curr Opin Psychiatry 2008; 21 (3): 260-267.

3. Karademir M, Sonmez MA, Akcilar R, Kocak E, Yay A, Eser O. Evaluation of therapeutic potential of intraperitoneal ozone gas in combination with insulin above cranial and spinal neuropathy in rats with diabetes mellitus. Bratisl Med J 2018; 119 (10): 636-641.

4. Akter K, Lanza EA, Martin SA, Myronyuk N, Rua M, Raffa RB. Diabetes mellitus and Alzheimer's disease: shared pathology and treatment? Br J Clin Pharmacol 2011; 71 (3): 365-376.

5. Craft S. Insulin resistance and Alzheimer's disease pathogenesis: potential mechanisms and implications for treatment. Curr Alzheimer Res 2007; 4 (2): 147-152.

6. Strachan MW, Deary IJ, Ewing FM, Frier BM. Is type II diabetes associated with an increased risk of cognitive dysfunction? A critical review of published studies. Diabetes Care 1997; 20 (3): 438-445.

7. Cukierman T, Gerstein HC, Williamson JD. Cognitive decline and dementia in diabetes-systematic overview of prospective observational studies. Diabetologia 2005; 48 (12): 2460-2469.

8. Vardy ER, Rice PJ, Bowie PC, Holmes JD, Grant PJ, Hooper NM. Increased circulating insulin-like growth factor-1 in late-onset Alzheimer's disease. J Alzheimer's Dis 2007; 12 (4): 285-290.

9. Kumar R, Chaterjee P, Sharma PK et al. Sirtuin1: A promising serum protein marker for early detection of Alzheimer's disease. PLoS One 2013: 8 (4): e61560.

10. Patel NV, Gordon MN, Connor KE et al. Caloric restriction attenuates Abeta-deposition in Alzheimer transgenic models. Neurobiol Aging 2005: 26 (7): 995-1000.

11. Guarente L. Mitochondria - a nexus for aging, calorie restriction, and sirtuins? Cell 2008; 132 (2): 171-176. 
812-816

12. Bonda DJ, Lee HG, Camins A et al. The sirtuin pathway in ageing and Alzheimer disease: mechanistic and therapeutic considerations. Lancet Neurol 2011; 10 (3): 275-279.

13. Julien C, Tremblay C, Emond V et al. Sirtuin 1 reduction parallels the accumulation of tau in Alzheimer disease. J Neuropathol Exp Neurol 2009; 68 (1): 48-58.

14. Fewlass DC, Noboa K, Pi-Sunyer FX, Johnston JM, Yan SD, Tezapsidis N. Obesity-related leptin regulates Alzheimer's Abeta. FASEB J 2004; 18 (15): 1870-1878.

15. Greco SJ, Bryan KJ, Sarkar S et al. Leptin reduces pathology and improves memory in a transgenic mause model of Alzheimer's disease. J Alzheimer's Dis 2010; 19 (4): 1155-1167.

16. Greco SJ, Sarkar S, Johnston JM et al. Leptin reduces Alzheimer's disease related tau phosphorylation in neuronal cells.Biochem and Biophys Res Commun 2008; 376 (3): 536-541.

17. McKhann G, Drachman DD, Folstein M, Katzman R, Price D, Stadlan EM. Clinical diagnosis of Alzheimer's disease: Report of the NNINCDS-ADRDA Work Group under the auspices of the Department of Health and Human Services Task Force on Alzheimer's Disease. Neurology, 1984; 34 (7): 939-944.

18. Bell CC. MDDSM-IV: Diagnostic and Statistical Manual of Mental Disorders. JAMA 1994; 272 (10): 828-829.

19. Folstein MF, Folstein SE, McHugh PR. Mini-Mental State: A practical method for grading the cognitive state of patients for clinician. J Psychiatr Res 1975; 12 (3): 189-198.

20. Güngen C, Ertan T, Eker E, Yaşar R, Engin F. The reliability and validity of the standardized Mini Mental Test Examination in the diagnosis of moderate dementia in Turkish population. Turk Psikiyatri Derg 2002; 13 (4): 273-281.
21. Johnston JM, Greco SJ, Hamzelou A, Ashford JW, Tezapsidis N. Repositioning leptin as a therapy for Alzheimer's disease. Therapy 2011; 8 (5): 481-490.

22. Sato N, Morishita R. Roles of vascular and metabolic components in cognitive dysfunction of Alzheimer disease: short- and long-term modification by non-genetic risk factors. Front Aging Neurosci 2013; 5: 64.

23. Gasparini L, Netzer WJ, Greengard P, Xu H. Does insulin dysfunction play a role in Alzheimer's disease? Trends in Pharmacol Sci 2002; 23 (6): 288-293.

24. Trejo JL, Carro E, Lopez-Lopez C, Torres-Aleman I. Role of serum insulin-like growth factor I in mammalian brain aging. Growth Horm IGF Res 2004; 14 (Supll): 39-43.

25. Jianping L, Zhibing Y, Wei Q, Zhikai C, Jie X, Jinbiao L. Low avidity and level of serum anti-Abeta antibodies in Alzheimer disease. Alzheimer Dis Assoc Disord 2006; 20 (3): 127-132.

26. Yamamoto K, Shimada H, Koh H, Ataka S, Miki T. Serum levels of albumin-amyloid beta complexes are decreased in Alzheimer's disease. Geriatr Gerontol Int 2014; 14 (3): 716-723.

27. Lieb W, Beiser AS, Vasan RS et al. Association of plasma leptin levels with incident Alzheimer disease and MRI measures of brain aging. JAMA 2009; 302 (23): 2565-2572.

28. Tezapsidis N, Johnston JM, Smith MA et al. Leptin: a novel therapeutic strategy for Alzheimer's disease. J Alzheimer's Dis 2009; 16 (4): 731-740.

29. Bigalke B, Schreitmüller B, Sopova K et al. Adipocytokines and CD34 progenitor cells in Alzheimer's disease. PLoS One 2011; 6 (5): e20286. 